University of Wollongong

Research Online

Faculty of Engineering and Information

Faculty of Engineering and Information

Sciences - Papers: Part A

Sciences

$1-1-1998$

Completeness of two systems of illative combinatory logic for first-order propositional and predicate calculus

\author{
Wil Dekkers
}

Martin Bunder

University of Wollongong, mbunder@uow.edu.au

Henk Barendregt

Radboud University Nijmegen

Follow this and additional works at: https://ro.uow.edu.au/eispapers

Part of the Engineering Commons, and the Science and Technology Studies Commons

Research Online is the open access institutional repository for the University of Wollongong. For further information contact the UOW Library: research-pubs@uow.edu.au 


\title{
Completeness of two systems of illative combinatory logic for first-order propositional and predicate calculus
}

\author{
Abstract \\ Illative combinatory logic consists of the theory of combinators or lambda calculus extended by extra \\ constants (and corresponding axioms and rules) intended to capture inference. The paper considers 4 \\ systems of illative combinatory logic that are sound for first-order propositional and predicate calculus.

\section{Keywords} \\ predicate, propositional, order, calculus, first, completeness, logic, combinatory, illative, systems, two \\ Disciplines \\ Engineering | Science and Technology Studies

\section{Publication Details} \\ Dekkers, W., Bunder, M. \& Barendregt, H. (1998). Completeness of two systems of illative combinatory \\ logic for first-order propositional and predicate calculus. Archive for Mathematical Logic, 37 (5-6), \\ 327-341.
}




\title{
Completeness of two systems of illative combinatory logic for first-order propositional and predicate calculus
}

\author{
Wil Dekkers ${ }^{1}$, Martin Bunder ${ }^{2}$, Henk Barendregt ${ }^{1}$ \\ ${ }^{1}$ Faculty of Mathematics and Computer Science, Catholic University, NL-6525 ED Nijmegen, The \\ Netherlands (e-mail: wil@cs.kun.nl, henk@cs.kun.nl) \\ ${ }^{2}$ Faculty of Informatics, Department of Mathematics, University of Wollongong, NSW Australia \\ (e-mail: Martin_Bunder@uow.edu.au)
}

\begin{abstract}
Illative combinatory logic consists of the theory of combinators or lambda calculus extended by extra constants (and corresponding axioms and rules) intended to capture inference. The paper considers 4 systems of illative combinatory logic that are sound for first-order propositional and predicate calculus. The interpretation from ordinary logic into the illative systems can be done in two ways: following the propositions-as-types paradigm, in which derivations become combinators, or in a more direct way, in which derivations are not translated. Both translations are closely related in a canonical way. In a preceding paper, Barendregt, Bunder and Dekkers, 1993, we proved completeness of the two direct translations. In the present paper we prove completeness of the two indirect translations by showing that the corresponding illative systems are conservative over the two systems for the direct translations. In another version, DBB (1997), we shall give a more direct completeness proof. These papers fulfill the program of Church and Curry to base logic on a consistent system of $\lambda$-terms or combinators. Hitherto this program had failed because systems of ICL were either too weak (to provide a sound interpretation) or too strong (sometimes even inconsistent).
\end{abstract}

\section{Introduction}

The theory of combinators and the lambda calculus are theories that succesfully analyze the notion of effective computability. However, the original founders of these subjects, Curry and Church, also had as aim to provide a basis for 
logic (and thereby mathematics). Unfortunately, it was shown by Kleene and Rosser that their systems were inconsistent. Curry and his school then started a program of defining several systems of illative combinatory logic (ICL) of varying strength. The goal was to find stronger and stronger systems which were consistent and weaker and weaker systems which were inconsistent but strong enough to interpret logic, hoping to end up with a consistent system in which logic could be interpreted.

Following this methodology, Bunder introduced restrictions on the rules of the illative constants so that first-order propositional and predicate calculus could be interpreted in the resulting systems. In all his systems the usual derivation of Curry's paradox is blocked, but the consistency of the systems remains an open question.

In BBD (1993) (Barendregt, Bunder and Dekkers, 1993) we introduced 4 systems of illative combinatory logic. We derived roughly the following soundness result

$$
\Delta \vdash_{\mathrm{L}} A \Rightarrow[\Delta] \vdash_{\mathrm{C}}[A],
$$

where $\mathrm{L}$ represents propositional or predicate logic and [-] one of two possible translations of each system into an ICL system C. Of the interpretations one is the propositions-as-types interpretation due to Curry, Howard and de Bruijn, the other is a more direct interpretation.

We derived completeness results for the direct translations of propositional and predicate calculus into 2 of the 4 systems of ICL. These, again roughly, took the following form

$$
[\Delta] \vdash_{\mathrm{C}}[A] \Rightarrow \Delta \vdash_{\mathrm{L}} A \text {. }
$$

In the present paper we shall prove that also the two indirect translations are complete.

These completeness results imply the consistency of the ICL's involved.

\section{Summary of results in preceding and present paper}

This paper is a continuation of a preceding paper, BBD (1993), by the same authors. We will refer to that paper most of the time simply as B; so for example Proposition B2.11 means Proposition 2.11 in BBD (1993). As the present paper is a continuation of that paper, we will not repeat all the definitions and results here but will give only a very short summary. For an introduction into ICL-systems, motivations, examples, propositions with proofs, and references we refer to BBD (1993).

We had as aim in the preceding paper to prove

(i) $\Delta \vdash_{\text {PROP }} \varphi \Leftrightarrow \forall$ closed $r\left[[\Delta]_{r}^{1}, \Gamma_{r}^{1}(\Delta, \varphi) \vdash_{\mathscr{T P}}[\varphi]_{r}^{1}\right]$;

(ii) $\Delta \vdash_{\text {PRED }} \varphi \Leftrightarrow \forall$ closed $r\left[\Gamma_{r, s}^{1,+},[\Delta]_{r}^{1}, \Gamma(\Delta, \varphi) \vdash_{\mathscr{T} \Xi}[\varphi]_{r}^{1}\right]$;

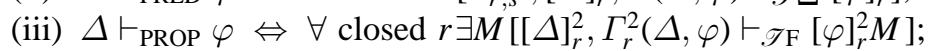

(iv) $\Delta \vdash_{\text {PRED }} \varphi \Leftrightarrow \forall$ closed $r \exists M\left[\Gamma_{r, s}^{2,+},[\Delta]_{r}^{2}, \Gamma(\Delta, \varphi) \vdash_{\mathscr{T}_{\mathrm{G}}}[\varphi]_{r}^{2} M\right]$. 
The proofs of soundness ( $\Rightarrow$ )were easy. (cf. Propositions B2.11 and B2.14). In Propositions B3.14 and B3.11 we proved completeness for the 2 direct translations []$^{1}: \mathrm{PROP} \rightarrow \mathscr{T P}$ and []$^{1}:$ PRED $\rightarrow \mathscr{T} \boldsymbol{\Xi},((\mathrm{i}) \Leftarrow$ and $(\mathrm{ii}) \Leftarrow)$, by specialising to $r=\mathrm{I}$ i.e.

$$
\begin{gathered}
{[\Delta]_{\mid}^{1}, \Gamma_{\mid}^{1}(\Delta, \varphi) \vdash_{\mathscr{T P}}[\varphi]_{\mid}^{1} \Rightarrow \Delta \vdash_{\mathrm{PROP}} \varphi \quad \text { in B3.14 }} \\
\Gamma_{\mid, s}^{1,+},[\Delta]_{\mid}^{1}, \Gamma(\Delta, \varphi) \vdash_{\mathscr{T} \Xi}[\varphi]_{\mid}^{1} \Rightarrow \Delta \vdash_{\mathrm{PRED}} \varphi \quad \text { in B3.11. }
\end{gathered}
$$

In the present paper we prove completeness for the 2 indirect translations [ $]^{2}$ : PROP $\rightarrow \mathscr{T F}$ and []$^{2}:$ PRED $\rightarrow \mathscr{T G},(($ iii $) \Leftarrow$ and $($ iv $) \Leftarrow)$, by specialising to $r=\mathrm{K}$. i.e.

$$
\begin{gathered}
{[\Delta]_{\mathrm{K}}^{2}, \Gamma_{\mathrm{K}}^{2}(\Delta, \varphi) \vdash_{\mathscr{F}}[\varphi]_{\mathrm{K}}^{2} M \Rightarrow \Delta \vdash_{\mathrm{PROP}} \varphi} \\
\Gamma_{\mathrm{K}, s}^{2,+},[\Delta]_{\mathrm{K}}^{2}, \Gamma(\Delta, \varphi) \vdash_{\mathscr{T} \mathrm{G}}[\varphi]_{\mathrm{K}}^{2} M \Rightarrow \Delta \vdash_{\mathrm{PRED}} \varphi .
\end{gathered}
$$

The completeness proof for the direct translation []$^{1}:$ PRED $\rightarrow \mathscr{T} \Xi((\mathrm{ii}) \Leftarrow)$ was given in the preceding paper in the following way. We had to show

$$
\Gamma^{\prime} \vdash \mathscr{T} \Xi[\varphi]_{1}^{1} \Rightarrow \Delta \vdash_{\text {PRED }} \varphi,
$$

where $\Gamma^{\prime}=\Gamma_{1, s}^{1,+},[\Delta]_{1}^{1}, \Gamma(\Delta, \varphi)$. First we defined in B3.2 a set $\mathscr{O}$ in order to analyze the terms $M$ such that $\Gamma^{\prime} \vdash_{\mathscr{T} \Xi} M$. We defined $\overline{\mathscr{O}}=\{M|\exists N \in \mathscr{O}|$ $\left.N={ }_{\beta \eta} M\right\}$ and it was clear that $\Gamma^{\prime} \subseteq \overline{\mathcal{O}}$. We showed in Proposition B3.10 that $\mathscr{O}$ is an invariant (modulo $\beta \eta$-equality) for derivations in $\mathscr{T} \boldsymbol{\Xi}$ :

$$
\Gamma \vdash_{\mathscr{T} \Xi} M, \Gamma \subseteq \overline{\mathscr{O}} \Rightarrow M \in \overline{\mathcal{O}} .
$$

Using this result we then proved in Proposition B3.11

$$
\Gamma^{\prime} \vdash_{\mathscr{T} \Xi}[\varphi]_{1}^{1} \Rightarrow \Delta \vdash_{\mathrm{PRED}} \varphi .
$$

The completeness proof for []$^{1}: \mathrm{PROP} \rightarrow \mathscr{T P}((\mathrm{i}) \Leftarrow)$ was done in a similar but easier way.

In the present paper we will prove completeness of []$^{2}:$ PROP $\rightarrow \mathscr{T F}$ and []$^{2}:$ PRED $\rightarrow \mathscr{T G}(($ iii $) \Leftarrow$ and (iv) $\Leftarrow)$, by specialising to $r=\mathrm{K}$.

Completenes of $\mathscr{T F}$ for PROP is relatively easy. We must show

$$
[\Delta]_{\mathrm{K}}^{2}, \Gamma_{\mathrm{K}}^{2}(\Delta, \varphi) \vdash_{\mathscr{T} \mathrm{F}}[\varphi]_{\mathrm{K}}^{2} M \Rightarrow \Delta \vdash_{\mathrm{PROP}} \varphi,
$$

i.e. (by Proposition B2.15: $\mathrm{K}[\varphi]_{r}^{1}=[\varphi]_{\mathrm{K} \circ r}^{2}$ )

$$
[\Delta]_{1}^{1}, \Gamma_{1}^{1}(\Delta, \varphi) \vdash_{\mathscr{F}}[\varphi]_{1}^{1} \Rightarrow \Delta \vdash_{\mathrm{PROP}} \varphi \text {. }
$$

Let us denote the grammar for the completeness proof of $\mathscr{T P}$ for PROP (defined in B3.12) by $\mathscr{Q}_{0}$ in order to distinguish it from $\mathscr{O}$ above. We shall prove in Proposition 3.3

$$
\Gamma \vdash_{\mathscr{F} \mathrm{F}} M, \Gamma \subseteq \overline{\mathscr{C}_{0}} \Rightarrow \Gamma \vdash_{\mathscr{T P}} M .
$$

Then completenes of $\mathscr{T F}$ for PROP for the second interpretation follows directly from completenes of $\mathscr{T P}$ for PROP for the first interpretation. 
Completeness of $\mathscr{T G}$ for PRED is a little bit more complicated. Again by Proposition B2.15 $\left(\mathrm{K}[\varphi]_{r}^{1}=[\varphi]_{\mathrm{K} \text { or }}^{2}\right)$, we see that we must prove

$$
\Gamma_{\mathrm{l}, s}^{1,+},[\Delta]_{1}^{1}, \Gamma(\Delta, \varphi) \vdash_{\mathscr{T}_{\mathrm{G}}}[\varphi]_{1}^{1} \Rightarrow \Delta \vdash_{\mathrm{PRED}} \varphi .
$$

We define a grammar, denoted by $\mathscr{Q}_{G}$ to distinguish it from $\mathscr{Q}$, and we will show in Proposition 4.11 that $\overline{\mathscr{O}_{G}}$ is closed under derivations in $\mathscr{T G}$ :

$$
\Gamma \vdash_{\mathscr{T}} M, \Gamma \subseteq \overline{\mathscr{C}_{G}} \Rightarrow M \in \overline{\mathscr{O}_{G}} .
$$

The invariant $\mathscr{O}_{G}$ is more complicated than $\mathscr{O}$ of B3.2 and we cannot use it to prove completeness directly in a way similar to the proof of completeness for $\mathscr{T} \Xi$ relative to PRED by means of $\mathscr{O}$ in B3.11. In fact we shall use $\mathscr{C}_{G}$ in order to show in Lemma 4.19 that in a proof of $\Gamma \vdash_{\mathscr{G}} M$, where $\Gamma \subseteq \overline{\mathcal{Q}}$, all cut formulas can be eliminated. From this we get in Proposition 4.23 conservativity of $\mathscr{T} G$ over $\mathscr{T} \Xi$ :

$$
\Gamma \subseteq \overline{\mathscr{O}}, M \in \overline{\mathscr{O}}, \Gamma \vdash_{\mathscr{T} \mathrm{G}} M \Rightarrow \Gamma \vdash_{\mathscr{T} \Xi} M
$$

and then completeness of $\mathscr{T G}$ for PRED is an immediate corollary of this proposition and Proposition B3.11 (completeness of $\mathscr{T} \Xi$ for PRED).

2.1. Remark. In the present paper we give the completeness proofs of $\mathscr{T F}$ for PROP and $\mathscr{T G}$ for PRED by specialising to $r=\mathrm{K}$. One may wonder if the proofs can be given for other $r$, especially for $r=\mathrm{I}$. This is indeed the case. In Sect. 5 we will comment on that.

\section{Completeness of $\mathscr{T F}$ for PROP}

We must show for $\varphi \in \mathbb{F}_{\mathrm{PROP}}, \Delta \subseteq \mathbb{F}_{\mathrm{PROP}}$ :

$$
\forall \text { closed } r \exists M \in \Lambda\left[[\Delta]_{r}^{2}, \Gamma_{r}^{2}(\Delta, \varphi) \vdash_{\mathscr{F}_{\mathrm{F}}}[\varphi]_{r}^{2} M\right] \Rightarrow \Delta \vdash_{\mathrm{PROP}} \varphi .
$$

It is sufficient to show

$$
\exists M \in \Lambda\left[[\Delta]_{\mathrm{K}}^{2}, \Gamma_{\mathrm{K}}^{2}(\Delta, \varphi) \vdash_{\mathscr{T}}[\varphi]_{\mathrm{K}}^{2} M\right] \Rightarrow \Delta \vdash_{\mathrm{PROP}} \varphi .
$$

We have by Proposition B2.15 the following relation between the two interpretations:

$$
\mathrm{K}[\varphi]_{r}^{1}=[\varphi]_{\mathrm{Kor}}^{2} .
$$

Hence we must prove

$$
[\Delta]_{1}^{1}, \Gamma_{1}^{1}(\Delta, \varphi) \vdash_{\mathscr{F} \mathrm{F}}[\varphi]_{1}^{1} \Rightarrow \Delta \vdash_{\mathrm{PROP}} \varphi .
$$

We omit I in the notation and we prove

$$
[\Delta]^{1}, \Gamma^{1}(\Delta, \varphi) \vdash_{\mathscr{T}}[\varphi]^{1} \Rightarrow \Delta \vdash_{\mathrm{PROP}} \varphi .
$$


$(*)$ could be proved in exactly the same way as Proposition B3.14 (Completeness for $\mathscr{T P}$ relative to $\mathrm{PROP}$ ), changing only $\mathscr{T P}$ in $\mathscr{T F}$. The reason for this is the following. In the proof of Proposition B2.7 we showed that $P_{e}, P_{i}$ and $P_{H}$ follow from $\mathrm{F}_{\mathrm{e}}, \mathrm{F}_{\mathrm{i}}$ and $\mathrm{F}_{\mathrm{L}}$ by the substitutions of $\mathrm{K} X$ for $X$ and $\mathrm{K} Y$ for $Y$. An inspection of the proof of completeness for $\mathscr{T P}$ relative to PROP shows that every $X$ and $Y$ occurring in the proof in cases $\mathrm{P}_{\mathrm{e}}, \mathrm{P}_{\mathrm{i}}$ and $\mathrm{P}_{\mathrm{H}}$ have the form $\mathrm{K} X_{1}$ and $\mathrm{K} Y_{1}$ and therefore also a substitution in the opposite direction is possible.

We will give another proof and show that $(*)$ is a consequence of Proposition B3.14 by proving in Proposition 3.3 that we have

$$
[\Delta]^{1}, \Gamma^{1}(\Delta, \varphi) \vdash_{\mathscr{F} \mathrm{F}}[\varphi]^{1} \Rightarrow[\Delta]^{1}, \Gamma^{1}(\Delta, \varphi) \vdash_{\mathscr{T} P}[\varphi]^{1} .
$$

3.1. Definition If $\mathscr{R}$ is any grammatical class in this paper then

$$
\overline{\mathscr{R}}=\left\{X \mid(\exists Y) Y \in \mathscr{R} \wedge X=_{\beta \eta} Y\right\} .
$$

3.2. Definition (Grammar for derivable formulas for $\mathscr{T F}$ ) We copy Definition B3.12, adding the index 0 .

$$
\begin{aligned}
& \mathscr{R}=\mathscr{T} \mid \mathscr{R} \supset \mathscr{R} \equiv \Xi(\mathrm{K} \mathscr{R})(\mathrm{K} \mathscr{R}) ; \\
& \mathscr{G}_{0}=\mathrm{H} \mathscr{B} \mid \mathscr{R} \supset \mathscr{O}_{0} \equiv \mathbf{\Xi}(\mathrm{K} \mathscr{O})\left(\mathrm{K} \mathscr{G}_{0}\right) ; \\
& \mathscr{C}_{0}=\mathscr{R}_{0} \mid \mathscr{R} \text {. }
\end{aligned}
$$

Note that $[\Delta]^{1}, \Gamma^{1}(\Delta, \varphi) \subseteq \overline{\mathscr{C}_{0}}$.

3.3. Proposition $\mathscr{T F}$ is conservative over $\mathscr{T P}$ in the following sense

$$
\Gamma \vdash_{\mathscr{T}_{\mathrm{F}}} M \& \Gamma \subseteq \overline{\mathscr{C}_{0}} \Rightarrow \Gamma \vdash_{\mathscr{T} \mathrm{P}} M \& M \in \overline{\mathscr{O}_{0}} .
$$

Proof. We rewrite the rules for $\mathscr{T} \mathrm{F}$ in the following way:

$$
\begin{array}{llll}
\mathrm{F}_{\mathrm{e}} & \Gamma \vdash \Xi X(Y \circ Z), \quad \Gamma \vdash X V & \Rightarrow & \Gamma \vdash(Y \circ Z) V ; \\
\mathrm{F}_{\mathrm{i}} & \Gamma, X x \vdash(Y \circ Z) x, \quad \Gamma \vdash \mathrm{LX}, x \notin \mathrm{FV}(\Gamma, X, Y, Z) & \Rightarrow & \Gamma \vdash \Xi X(Y \circ Z) ; \\
\mathrm{F}_{\mathrm{L}} & \Gamma, X x \vdash \mathrm{L} Y, \quad \Gamma \vdash \mathrm{L} X, \quad x \notin \mathrm{FV}(\Gamma, X, Y) & \Rightarrow & \Gamma \vdash \mathrm{L}(\mathrm{FX} Y) .
\end{array}
$$

The proof of (**) is by induction on the proof of $\Gamma \vdash_{\mathscr{F}_{\mathrm{F}}} M$.

Case $\mathrm{F}_{\mathrm{e}} . \Gamma \vdash_{\mathscr{T}_{\mathrm{F}}} M$ is $\Gamma \vdash_{\mathscr{T}_{\mathrm{F}}}(Y \circ \mathrm{Z}) V$ as consequence of

$$
\Gamma \vdash_{\mathscr{T} F} \Xi X(Y \circ Z), \quad \Gamma \vdash_{\mathscr{T} F} X V \text {. }
$$

By the induction hypothesis one has

$$
\Gamma \vdash_{\mathscr{T} \mathrm{P}} \Xi X(Y \circ Z), \quad \Gamma \vdash_{\mathscr{T} \mathrm{P}} X V,
$$




$$
\boldsymbol{\Xi} X(Y \circ \mathrm{Z})=\boldsymbol{\Xi}(\mathrm{K} p)(\mathrm{K} O), \quad X V=p,
$$

where $p \in \mathscr{O}$ and $O \in \mathscr{C}_{0}$. By rule $\mathrm{P}_{\mathrm{e}}$ we have $\Gamma \vdash_{\mathscr{T}} O$, where $O=(Y \circ Z) V=$ $M$.

Case $\mathrm{F}_{\mathrm{i}} . \Gamma \vdash_{\mathscr{F}_{\mathrm{F}}} M$ is $\Gamma \vdash_{\mathscr{T} \mathrm{F}} \Xi X(Y \circ Z)$ as consequence of $\Gamma \vdash_{\mathscr{T}_{\mathrm{F}}} \mathrm{L} X, \quad \Gamma, X x \vdash_{\mathscr{F}_{\mathrm{F}}}(Y \circ Z) x$ with $x \notin \mathrm{FV}(\Gamma, X, Y, Z)$.

By the IH we have

$$
\Gamma \vdash_{\mathscr{T} \mathrm{P}} \mathrm{L} X, X=\mathrm{K}_{p}, \quad \Gamma, p \vdash_{\mathscr{T} \mathrm{P}}(Y \circ Z) x
$$

and $(Y \circ Z) x=O \in \mathscr{C}_{0}$. Now $x \notin \mathrm{FV}(\Gamma, p)$, so $x \notin \mathrm{FV}(O)$ by Proposition B3.10, hence $Y \circ Z=\mathrm{K} O$. Therefore by rule $\mathrm{P}_{\mathrm{i}}$ we get

$$
\Gamma \vdash_{\mathscr{T} \mathrm{P}} p \supset O
$$

and $p \supset O=\Xi(\mathrm{K} p)(\mathrm{K} O)=\Xi X(Y \circ Z)=M$.

Case $\mathrm{F}_{\mathrm{L}} . \Gamma \vdash_{\mathscr{T}} M$ is $\Gamma \vdash_{\mathscr{T}} \mathrm{L}(\mathrm{F} X Y)$ as consequence of $\Gamma \vdash_{\mathscr{F}} \mathrm{L} X, \quad \Gamma, X x \vdash_{\mathscr{F}} \mathrm{L}(Y)$ with $x \notin \mathrm{FV}(\Gamma, X, Y)$.

By the IH one has

$$
\Gamma \vdash_{\mathscr{P} \mathrm{P}} \mathrm{L} X, \quad X=\mathrm{K} p, \quad \Gamma, p \vdash_{\mathscr{T}} \mathrm{L}(Y), \quad Y=\mathrm{K}_{1} .
$$

Hence by rule $\mathrm{P}_{\mathrm{H}} \quad \Gamma \vdash_{\mathscr{P}} \mathrm{H}\left(p \supset p_{1}\right)$. Now $\mathrm{H}\left(p \supset p_{1}\right)=\mathrm{L}\left(\mathrm{K}\left(p \supset p_{1}\right)\right)=$ $\mathrm{L}\left(\mathrm{F}(\mathrm{K} p)\left(\mathrm{K}_{1}\right)\right)=\mathrm{L}(\mathrm{F} X Y)$. So $\Gamma \vdash_{\mathscr{T}} \mathrm{P} M$.

\section{Completeness of $\mathscr{T G}$ for PRED}

We will show for $\varphi \in \mathbb{F}_{\text {PRED }}, \Delta \subseteq \mathbb{F}_{\mathrm{PRED}}$ :

$$
\forall \text { closed } r\left[\Gamma_{r, s}^{2,+},[\Delta]_{r}^{2}, \Gamma(\Delta, \varphi) \vdash_{\mathscr{T G}}[\varphi]_{r}^{2} M \text { some } M\right] \Rightarrow \Delta \vdash_{\text {PRED }} \varphi .
$$

It is sufficient to show

$$
\Gamma_{\mathrm{K}, s}^{2,+},[\Delta]_{\mathrm{K}}^{2}, \Gamma(\Delta, \varphi) \vdash_{\mathscr{T}_{\mathrm{G}}}[\varphi]_{\mathrm{K}}^{2} M \Rightarrow \Delta \vdash_{\mathrm{PRED}} \varphi,
$$

i.e. (by Proposition B2.15: $\mathrm{K}[\varphi]_{r}^{1}=[\varphi]_{\mathrm{Ko},}^{2}$ )

$$
\Gamma_{s}^{1,+},[\Delta]^{1}, \Gamma(\Delta, \varphi) \vdash_{\mathscr{T} \mathrm{G}}[\varphi]^{1} \Rightarrow \Delta \vdash_{\mathrm{PRED}} \varphi .
$$

Here $\Gamma_{s}^{1,+},[\Delta]^{1}$ and $[\varphi]^{1}$ stand for $\Gamma_{1, s}^{1,+},[\Delta]_{\mid}^{1}$ and $[\varphi]_{\mid}^{1}$.

The proof is again in several steps. First we define in 4.2 an invariant $\mathscr{Q}_{G}$ (modulo $\beta \eta$-equality) for derivations in $\mathscr{T G}$. Using this invariant we show in Lemma 4.19 that in a proof of $\Gamma_{s}^{1,+},[\Delta]^{1}, \Gamma(\Delta, \varphi) \vdash_{\mathscr{T}_{\mathrm{G}}} N$ all cuts can be eliminated. From this we get in Proposition 4.23

$$
\Gamma_{s}^{1,+},[\Delta]^{1}, \Gamma(\Delta, \varphi) \vdash_{\mathscr{T G}} N \Rightarrow \Gamma_{s}^{1,+},[\Delta]^{1}, \Gamma(\Delta, \varphi) \vdash_{\mathscr{T} \Xi} N
$$


and then completeness of $\mathscr{T G}$ for PRED is an immediate corollary of this proposition and Proposition B3.11. Instead of $\vdash_{\mathscr{G}}$ we shall mostly write $\vdash$.

Now first we repeat Definition B3.2, of the invariant $\mathscr{O}$ for derivations in $\mathscr{T} \Xi$ for the manysorted structure of our example, in order to contrast it with the one for derivable statements in $\mathscr{T} \mathrm{G}$ of Definition 4.2 and also because we need it in the sequel. Instead of $x_{i}, \mathscr{T}_{i}$ and $\mathscr{T}_{i}$ we shall write $x^{A_{i}}, \mathscr{T}^{A_{i}}$ and $\mathscr{T}^{A_{i}}$.

4.1. Definition (Grammar for derivable statements in $\mathscr{T} \Xi$ )

$$
\begin{aligned}
& \mathscr{T}=\mathscr{T}^{A_{1}} \mid \mathscr{T}^{A_{2}} \\
& \mathscr{T}^{A_{1}}=\mathscr{V}^{A_{1}}|a| f \mathscr{T}^{A_{1}} \mid g \mathscr{T}^{A_{1}} \cdot \mathscr{T}^{A_{2}} ; \\
& \mathscr{T}^{A_{2}}=\mathscr{V}^{A_{2}} ; \\
& \mathscr{P}=P \mathscr{T}^{A_{1}}\left|\boldsymbol{\Xi} A_{i}\left(\lambda x^{A_{i}} \mathscr{\mathscr { A }}\right)\right| \boldsymbol{\Xi}(\mathrm{K} \mathscr{\mathscr { A }}(\mathrm{K} \mathscr{\mathscr { P }}) ; \\
& \mathscr{G}=\mathrm{L} A_{i}\left|A_{i} \mathscr{T}^{A_{i}}\right| \Xi A_{i}\left(\lambda x^{A_{i}} \mathscr{\mathscr { S }}\right) \mid \boldsymbol{\Xi}(\mathrm{K} \mathscr{\mathscr { P }}(\mathrm{K} \mathscr{\mathscr { T }}) \mid \mathrm{L}(\mathrm{K} \mathscr{H}) \\
& \mathscr{O}=\mathscr{O} \mid \mathscr{P} \text {. }
\end{aligned}
$$

Now we will define the invariant $\mathscr{C}_{G}$ for derivable statements in $\mathscr{T G}$. $\mathscr{Q}_{G}$ is more complicated then the grammar $\mathscr{O}$ for $\mathscr{T} \boldsymbol{\Xi}$ for several reasons:

Write $\Gamma^{\prime}$ for $\Gamma_{s}^{1,+},[\Delta]^{1}, \Gamma(\Delta, \varphi)$. If $\Gamma^{\prime} \vdash \mathrm{LX}$ in system $\mathscr{T} \boldsymbol{\Xi}$ then $X=A_{i}$ or $X=\mathrm{K} p$ with $p \in \mathscr{P}$. But if $\Gamma^{\prime} \vdash \mathrm{L} X$ in system $\mathscr{T} \mathrm{G}$ then we can have for example $X=\mathrm{F} A_{1} A_{2}$ or even $X=\mathrm{F}(\mathrm{K} p) A_{2}$. Therefore we define an extension $\mathscr{C}_{G}$ of the set of sorts $\left\{A_{1}, A_{2}\right\}$ containing these $X^{\prime}$ s. We need for each $l \in \mathscr{C}_{G}$ a set of variables $\mathscr{T}_{G}^{l}$, similar to the sets $\mathscr{V}^{A_{1}}$ and $\mathscr{V}^{A_{2}}$. If we took a different set of variables for each $l \in \mathscr{C}_{G}$, then the substitution lemma 4.5 , that is needed in the proof of Proposition 4.11, would not hold. Therefore we define for each $l \in \mathscr{D}_{G}$ the skeleton $\operatorname{sk}(l)$, such that $s k(\mathrm{~K} p)$ is the same for all $p \in \mathscr{P}_{G}$ and we define $\mathscr{T}_{G}^{l_{1}}=\mathscr{T}_{G}^{l_{2}}$ iff $s k\left(l_{1}\right)=s k\left(l_{2}\right)$.

The sets $\mathscr{T}_{G}^{l}$ will contain $\lambda$-abstractions: if $l=\mathrm{Fl}_{1} l_{2}$ and $t^{l_{2}} \in \mathscr{T}_{G}^{l_{2}}$ then $\lambda x^{l_{1}} \cdot t^{l_{2} \in \mathscr{T}_{G}}{ }^{l}$. This may seem a little bit strange, but in fact it is very natural. For example we have $\vdash \mathrm{F} A_{1} A_{1}\left(\lambda x^{A_{1}} \cdot x^{A_{1}}\right)$ by rule $\mathrm{F}_{\mathrm{i}}$. Hence $\lambda x^{A_{1}} \cdot x^{A_{1}}$ should be in $\mathscr{T}_{G}{ }^{F A_{1} A_{1}}$. Moreover in the proof of Lemma 4.9 we really need that $\lambda x^{l_{1}} \cdot t^{l_{2} \in \mathscr{T}_{G}} \mathrm{Fl}_{1} l_{2}$.

Finally $\mathscr{T}_{G}, \mathscr{G}_{G}$ and $\mathscr{O}_{G}$ are very similar to $\mathscr{P}, \mathscr{G}$ and $\mathscr{O}$, except that besides $\boldsymbol{\Xi}(\mathrm{K} p)(\mathrm{K} O) \in \mathscr{C}_{G}$ we now also have $\boldsymbol{\Xi}(\mathrm{K} p)\left(\lambda x^{\mathrm{K} p} . O\right) \in \mathscr{C}_{G}$, where $x^{\mathrm{K} p}$ may occur free in $O$.

4.2. Definition (Invariant $\mathscr{O}_{G}$ for derivable statements in $\mathscr{T} \mathrm{G}$ ) (i) $\Lambda^{s}(\boldsymbol{\Xi}, \mathrm{L})$ is, as in Definition B2.12, $\Lambda(\boldsymbol{\Xi}, \mathrm{L})$ extended by the extra constants $A_{1}, A_{2}, P, f, g, a$ associated with the signature s of the manysorted structure of our example.

(ii) Now we will define simultaneously sets $\mathscr{Q}_{G}$ and $\mathscr{D}_{G}$ of sorts (extensions of the set $\left.\left\{A_{1}, A_{2}\right\}\right)$, for each $l \in \mathscr{C}_{G}$ the skeleton sk( $(l)$, a set of variables $\mathscr{T}_{G}^{l}$ and a set of terms $\mathscr{T}_{G}^{l}$, sets $\mathscr{P}_{G}, \mathscr{G}_{G}$ and $\mathscr{O}_{G}$. 
Definition of $\mathscr{Q}_{G}$ and $\mathscr{L}_{G}$.

$$
\begin{aligned}
& \mathscr{Q}_{G}=A_{1}\left|A_{2}\right| \mathrm{G} l\left(\lambda x^{l} \cdot q\right) \quad\left(l \in \mathscr{C}_{G}, x^{l} \in \mathscr{T}_{G}^{l}, q \in \mathscr{Q}_{G}\right) ; \\
& \mathscr{C}_{G}=\mathscr{Q}_{G} \cup \mathrm{K} \mathscr{P}_{G} .
\end{aligned}
$$

Definition of $s k(l)$.

$$
\begin{aligned}
& s k\left(A_{i}\right)=A_{i} ; \\
& s k(\mathrm{~K} p)=\mathrm{K}\left(P x_{1}^{A_{1}}\right) \quad(\text { i.e. } s k(\mathrm{~K} p) \text { is independent of } p) ; \\
& s k\left(\mathrm{G} l\left(\lambda x^{l} \cdot q\right)\right)=\mathrm{G}(s k(l))\left(\lambda x^{l} \cdot s k(q)\right) .
\end{aligned}
$$

Definition of $\mathscr{T}_{G}^{l}$.

We assume that for each skeleton $s k(l)$ there is a different set of variables

$$
\mathscr{T}_{G}^{s k(l)}=\left\{x^{s k(l)}, x_{1}^{s k(l)}, x_{2}^{s k(l)}, \ldots, y^{s k(l)}, y_{1}^{s k(l)}, y_{2}^{s k(l)}, \ldots\right\}
$$

and for each $l \in \mathscr{D}_{G}$ we define

$$
\mathscr{T}_{G}^{l}=\mathscr{T}_{G}^{s k(l)}, \quad x^{l}=x^{s k(l)}, x_{i}^{l}=x_{i}^{s k(l)}, \quad y^{l}=y^{s k(l)}, y_{i}^{l}=y_{i}^{s k(l)} .
$$

Definition of $\mathscr{T}_{G}^{l}$.

$$
\begin{aligned}
& \mathscr{T}_{G}^{l}=a\left|\mathscr{T}_{G}^{l}\right| \mathscr{T}_{G}^{\mathrm{Fl}_{1} l} \mathscr{T}_{G}^{l_{1}}, \quad \text { if } l \equiv A_{1} ; \\
& \mathscr{T}_{G}^{l}=\mathscr{T}_{G}^{l} \mid \mathscr{T}_{G}^{\mathrm{F} l_{1} l} \mathscr{T}_{G}^{l_{1}}, \quad \text { if } l \equiv A_{2} ; \\
& \mathscr{T}_{G}^{l}=f\left|\mathscr{T}_{G}^{l}\right| \mathscr{T}_{G}^{F} l_{1} l \mathscr{T}_{G}^{l_{1}} \mid \lambda x^{A_{1}} . \mathscr{T}_{G}^{A_{1}}, \quad \text { if } l \equiv \mathrm{F} A_{1} A_{1} \text {; } \\
& \mathscr{T}_{G}^{l}=g\left|\mathscr{T}_{G}^{l}\right| \mathscr{T}_{G}{ }^{l_{1} l} \mathscr{T}_{G}^{l_{1}} \mid \lambda x^{A_{1}} . \mathscr{T}_{G}{ }^{\mathrm{F}_{2} A_{1}} \text {, if } l \equiv \mathrm{F} A_{1}\left(\mathrm{~F} A_{2} A_{1}\right) \text {; } \\
& \mathscr{T}_{G}^{l}=\mathscr{T}_{G}^{l}\left|\mathscr{T}_{G}{ }^{\mathrm{G} l_{1}\left(\lambda x^{l_{1}} \cdot l\right)} \cdot \mathscr{T}_{G}^{l_{1}}\right| \lambda x^{l_{2}} \cdot \mathscr{T}_{G}^{l_{3}} \text {, if } l \equiv \mathrm{G} l_{2}\left(\lambda x^{l_{2}} \cdot l_{3}\right) \text {, } \\
& \mathscr{T}_{G}^{l}=\Lambda^{s}(\boldsymbol{\Xi}, \mathrm{L}), \quad \text { if } l \equiv \mathrm{K} p . \\
& l \notin\left\{\mathrm{F} A_{1} A_{1}, \mathrm{~F} A_{1}\left(\mathrm{~F} A_{2} A_{1}\right)\right\} \text {; }
\end{aligned}
$$

Definition of $\mathscr{P}_{G}, \mathscr{G}_{G}$ and $\mathscr{C}_{G}$.

$$
\begin{aligned}
& \mathscr{P}_{G}=P \mathscr{T}_{G}^{A_{1}} \mid \boldsymbol{\Xi} l\left(\lambda x^{l} . \mathscr{P}_{G}\right) ; \\
& \mathscr{G}_{G}=\mathrm{L} \mathscr{D}_{G}\left|A_{i} \mathscr{T}_{G}^{A_{i}}\right| \Xi l\left(\lambda x^{l} . \mathscr{G}_{G}\right) ; \\
& \mathscr{C}_{G}=\mathscr{P}_{G} \mid \mathscr{S}_{G} \text {. }
\end{aligned}
$$

(iii) Notation.

$$
\begin{aligned}
& q, q_{1}, q_{2}, q^{\prime}, \ldots \text { denote elements of } \mathscr{Q}_{G} ; \\
& l, l_{1}, l_{2}, l^{\prime}, \ldots \text { denote elements of } \mathscr{L}_{G} ; \\
& p, p_{1}, p_{2}, p^{\prime}, \ldots \text { denote elements of } \mathscr{P}_{G} .
\end{aligned}
$$

4.3. Remarks. (i) $\Gamma_{s}^{1,+},[\Delta]^{1}, \Gamma(\Delta, \varphi) \subseteq \overline{\mathscr{O}} \subseteq \overline{\mathscr{O}_{G}}$.

(ii) If $\mathrm{FV}(q)=\emptyset$ then $\mathrm{Gl}\left(\lambda x^{l} \cdot q\right)=_{\beta} \mathrm{Flq}$. In that case we will denote $\mathrm{G} l\left(\lambda x^{l} . q\right)$ usually by $\mathrm{F} l q$. In fact we did that already in the above definition.

(iii) The grammar in the definition is not context-free and this causes some inaccuracies in the notation. In the definition of $\mathscr{P}_{G}$ (and similarly at some other places) instead of $\boldsymbol{\Xi} l\left(\lambda x^{l} . \mathscr{P}_{G}\right)$ we should write $\boldsymbol{\Xi} \mathscr{C}_{G}\left(\lambda x^{\mathscr{C}_{G}} . \mathscr{P}_{G}\right)$, but then we 
do not know that these two $\mathscr{B}_{G}$ 's represent the same $l \in \mathscr{D}_{G}$. (We may not have $\boldsymbol{\Xi} l_{1}\left(\lambda x^{l_{2}} . \mathscr{P}_{G}\right)$.)

(iv) In the definition of $\mathscr{T}_{G}^{l}$ instead of the clause $\mathscr{T}_{G}^{\mathrm{G} l_{1}\left(\lambda x^{l_{1}}, l\right)} \mathscr{T}_{G}^{l_{1}}$ one might expect, in view of rule $\mathrm{G}_{\mathrm{e}}$ a clause $t_{1}^{\mathrm{G} l_{1}\left(\lambda x^{l_{1}} \cdot l^{\prime}\right)} t_{2}^{l_{1}}$ where $l=l^{\prime}\left[x^{l_{1}}:=t_{2}^{l_{1}}\right]$, but that does not make a difference because we have for $l \in \mathscr{B}_{G}$

$$
\mathscr{T}_{G}^{l\left[x^{l_{1}}:=t^{l_{1}}\right]}=\mathscr{T}_{G}^{l},
$$

as follows easily from

$$
\mathscr{T}_{G}^{l\left[x^{l_{1}}: t^{l_{1}}\right]}=\mathscr{T}_{G}^{l}
$$

in Lemma 4.5 .

4.4. Lemma. $l_{1}, l_{2} \in \mathscr{D}_{G} \Rightarrow \mathrm{G} l_{1}\left(\lambda x^{l_{1}} \cdot l_{2}\right) \in \mathscr{B}_{G}$.

Proof. It is clear if $l_{2} \in Q_{G}$. Now let $l_{2}=\mathrm{K} p$. Then $\mathrm{G} l_{1}\left(\lambda x^{l_{1}} \cdot l_{2}\right)=\lambda z . \Xi l_{1}\left(\mathrm{~S}\left(\lambda x^{l_{1}} \cdot \mathrm{K} p\right) z\right)=\lambda z . \Xi l_{1}\left(\lambda x^{l_{1}} \cdot p\right)=\mathrm{K}\left(\Xi l_{1}\left(\lambda x^{l_{1}} \cdot p\right)\right) \in \mathrm{K} \mathscr{G}_{G}$.

4.5. Lemma. Let $\mathscr{W}=\mathscr{Q}_{G}, \mathscr{C}_{G}, \mathscr{T}_{G}^{l}, \mathscr{P}_{G}, \mathscr{G}_{G}$, or $\mathscr{Q}_{G}$ and let $w \in \mathscr{W}, x^{l_{1}}$ $\in \mathscr{T}_{G}^{l_{1}}, t^{l_{1}} \in \mathscr{T}_{G}^{l_{1}}$, then

$$
w\left[x^{l_{1}}:=t^{l_{1}}\right] \in \mathscr{W},
$$

moreover if $\mathscr{W}=\mathscr{L}_{G}$ then

$$
\operatorname{sk}\left(w\left[x^{l_{1}}:=t^{l_{1}}\right]\right)=\operatorname{sk}(w)
$$

and hence

$$
\mathscr{T}_{G}^{w\left[x^{l_{1}}:=t^{l_{1}}\right]}=\mathscr{T}_{G}^{w}
$$

Proof. By induction on the structure of $w$. Instead of $x^{l_{1}}$ and $t^{l_{1}}$ we shall write $x$ and $t$.

Case $w \in \mathscr{Q}_{G}$.

$$
\begin{aligned}
& w=A_{i} . \text { This is trivially ok. } \\
& \begin{aligned}
w=\mathrm{G} l_{2}\left(\lambda y^{l_{2}} \cdot q\right) \text {. Then } w[x:=t]=\mathrm{G}\left(l_{2}[x:=t]\right)\left(\lambda y^{l_{2}} \cdot q[x:=t]\right)= \\
=\mathrm{G}\left(l_{2}[x:=t]\right)\left(\lambda y^{l_{2}[x:=t]} \cdot q[x:=t]\right)
\end{aligned}
\end{aligned}
$$

(by the second part of the IH for $l_{2}$ ) and this is in $Q_{G}$ by the first part of the IH for $l_{2}$ and $q$. The second part of the $\mathrm{IH}$ for $l_{2}$ and $q$ yields $s k(w[x:=t])=s k(w)$.

Case $w \in \mathscr{D}_{G}$.

This follows directly from the cases $w \in \mathscr{Q}_{G}, w \in \mathscr{T}_{G}$.

Case $w=t^{l} \in \mathscr{T}_{G}^{l}$.

$l=A_{1}$. Now $w=x_{1}^{A_{1}}, a$ or $t_{1}^{\mathrm{G} l_{1}\left(\lambda x_{1}^{l_{1}} \cdot A_{1}\right)} t_{2}^{l_{1}}$. All these cases are easy.

$l=A_{2}$. Easy.

$l=\mathrm{G} l_{2}\left(\lambda y^{l_{2}} \cdot q\right)$. Now $w=x_{1}^{l}, t_{1}^{\mathrm{G} l_{3}\left(\lambda x_{1}^{l_{3}} \cdot l\right)} t_{2}^{l_{3}}, \lambda y^{l_{2}} \cdot t_{1}^{q}, f$ or $g$. Easy again.

$l=\mathrm{K} p$. Then $w[x:=t] \in \Lambda=\mathscr{T}_{G}^{\mathrm{K} p}$. 
Case $w \in \mathscr{T}_{G}$.

$$
\begin{gathered}
w=P t_{1}^{A_{1}} \text {. Then } w[x:=t]=P\left(t_{1}^{A_{1}}[x:=t]\right) . \\
w=\boldsymbol{\Xi} l_{2}\left(\lambda y^{l_{2}} \cdot p\right) \text {. Then } w[x:=t]=\boldsymbol{\Xi}\left(l_{2}[x:=t]\right)\left(\lambda y^{l_{2}} \cdot p[x:=t]\right)= \\
=\boldsymbol{\Xi}\left(l_{2}[x:=t]\right)\left(\lambda y^{l_{2}[x:=t]} \cdot p[x:=t]\right) .
\end{gathered}
$$

Case $w \in \mathscr{G}_{G}$. Similarly.

4.6. Lemma. Let $c X_{1} \ldots X_{n}=\beta_{\beta \eta} M$ for some constant $c$ and some $M \in \mathscr{Q}_{G}$. Then $n \in\{1,2\}$ and $M \equiv c Y_{1} \ldots Y_{n}$ with $Y_{i}=_{\beta \eta} X_{i}$.

Proof. By Church-Rosser for $\beta \eta$-reduction.

4.7. Lemma. Let $q \in \mathscr{Q}_{G} p \in \mathscr{P}_{G}, M \in \Lambda^{s}(\boldsymbol{\Xi}, \mathrm{L})$ and let $\sigma$ substitute variables for variables. Then $q M \neq_{\beta \eta} p^{\sigma}$.

Proof. By an easy induction on $q$, using Church-Rosser.

In the definition of $\mathscr{G}_{G}$ one of the clauses is $A_{i} \mathscr{T}_{G}^{A_{i}}$ where one might expect the more general clause $q \mathscr{T}_{G}^{q}$. But $A_{i} \mathscr{T}_{G}^{A_{i}}$ is sufficient as follows from the next lemma.

\subsection{Lemma.}

$$
q t^{q} \in \overline{\mathscr{G}_{G}} \text { for each } q \in \mathscr{Q}_{G} \text {. }
$$

Proof. By induction on the structure of $q$. It is clear if $q=A_{1}$ or $A_{2}$. So let $q=\mathrm{G} l_{1}\left(\lambda x^{l_{1}} \cdot q_{1}\right)$. Then $q t^{q}=\mathrm{G} l_{1}\left(\lambda x^{l_{1}} \cdot q_{1}\right) t^{q}=\boldsymbol{\Xi} l_{1}\left(\mathrm{~S}\left(\lambda x^{l_{1}} \cdot q_{1}\right) t^{q}\right)=$ $\boldsymbol{\Xi} l_{1}\left(\lambda x^{l_{1}} \cdot q_{1}\left(t^{q} x^{l_{1}}\right)\right)$. Now $t^{q} \in \mathscr{T}_{G}^{q}=\mathscr{T}_{G}^{\mathrm{G} l_{1}\left(\lambda x^{l_{1}} \cdot q_{1}\right)}$ and $x^{l_{1} \in \mathscr{T}_{G}^{l_{1}}}$ hence $t^{q} x^{l_{1}} \in \mathscr{T}_{G}^{q_{1}}$. So by the induction hypothesis one has $q_{1}\left(t^{q} x^{l_{1}}\right) \in \overline{\mathscr{G}_{G}}$. Hence $\Xi l_{1}\left(\lambda x^{l_{1}} \cdot q_{1}\left(t^{q} x^{l_{1}}\right)\right)$ $\in \overline{\mathscr{G}_{G}}$.

\subsection{Lemma.}

$$
l V \in \overline{\sigma_{G}} \Rightarrow V \in \overline{\mathscr{T}_{G}^{l}} .
$$

Proof. By induction on the structure of $l$. It is clear if $l=A_{1}, A_{2}$ or $\mathrm{K} p$. So let $l=\mathrm{G} l_{1}\left(\lambda x^{l_{1}} \cdot q\right)$. Then $l V=\Xi l_{1}\left(\lambda x^{l_{1}} \cdot q\left(V x^{l_{1}}\right)\right)$. So $q\left(V x^{l_{1}}\right) \in \overline{\mathscr{O}_{G}}$ and hence by the induction hypothesis we have $V x^{l_{1}}=t^{q} \in \mathscr{T}_{G}^{q}$. So $V=\lambda x^{l_{1}} \cdot t^{q} \in \mathscr{T}_{G}^{l}$.

4.10. Remark. Clearly $(\mathrm{K} p) t^{\mathrm{K} p}=p \in \mathscr{P}_{G}$, hence combining Lemmas 4.8 and 4.9 we get

$$
l V \in \overline{\mathscr{O}_{G}} \Leftrightarrow V \in \overline{\mathscr{T}_{G}} .
$$

Now we will prove $\Gamma \vdash M, \Gamma \subseteq \overline{\mathscr{C}_{G}} \Rightarrow M \in \overline{\mathscr{O}_{G}}$. The proof is different from the proof of Proposition B3.10, because we do not have

$$
\Gamma \vdash M, \Gamma \subseteq \overline{\mathscr{O}_{G}} \& u \notin_{\beta \eta} \mathrm{FV}(\Gamma) \Rightarrow u \notin_{\beta \eta} \mathrm{FV}(M) .
$$

This is shown by the following example

$$
\mathrm{F}(\mathrm{K} p) A_{1} x, p \vdash A_{1}(x u) .
$$


In fact this is the reason that in the grammar $\mathscr{O}_{G}$ we have elements $\boldsymbol{\Xi}(\mathrm{K} p)\left(\lambda x^{\mathrm{K} p} . O\right)$ instead of the simpler elements $\boldsymbol{\Xi}(\mathrm{K} p)(\mathrm{K} O)$.

4.11. Proposition. In $\mathscr{T} \mathrm{G}$ we have

$$
\Gamma \vdash M, \Gamma \subseteq \overline{\sigma_{G}} \Rightarrow M \in \overline{\mathscr{O}_{G}} .
$$

Proof. By induction on the derivation of $\Gamma \vdash M$. We only consider the three specific rules $\Xi_{\mathrm{e}}, \Xi_{\mathrm{i}}$ and $\mathrm{G}_{\mathrm{L}}$. ( The two general rules are easy and $\mathrm{G}_{\mathrm{e}}, \mathrm{G}_{\mathrm{i}}$ are equivalent to $\Xi_{\mathrm{e}}, \Xi_{\mathrm{i}}$.)

Case $\Xi_{\mathrm{e}} . \Gamma \vdash M$ is $\Gamma \vdash Y V$ as direct consequence of $\Gamma \vdash \Xi X Y, \quad \Gamma \vdash X V$.

By the IH one has $\Xi X Y \in \overline{\mathscr{O}_{G}}, X V \in \overline{\mathscr{C}_{G}}$. From $\Xi X Y \in \overline{\mathscr{C}_{G}}$ it follows that $X=$ $l, Y=\lambda x^{l} . O$ where $O \in \mathscr{O}_{G}$. Now $X V=l V \in \overline{\mathscr{O}_{G}}$, hence $V=t^{l} \in \mathscr{T}_{G}^{l}$ by Lemma 4.9. $M=\left(\lambda x^{l} . O\right) t^{l}=O\left[x^{l}:=t^{l}\right] \in \mathscr{Q}_{G}$ by Lemma 4.5.

Case $\Xi_{\mathrm{i}} . \Gamma \vdash M$ is $\Gamma \vdash \Xi X Y$ as direct consequence of $\Gamma \vdash \mathrm{L} X, \quad \Gamma, X x \vdash Y x$ with $x \notin \mathrm{FV}(\Gamma, X, Y)$.

By the IH one has $\mathrm{L} X \in \overline{\mathscr{O}_{G}}$, hence $X=l \in \mathscr{D}_{G}$. Now $x$ is any variable, so we may assume that $x=x^{l} \in \mathscr{T}_{G}^{l}$. Then $l x \in \overline{\mathscr{O}_{G}}$ by Lemma 4.8 , hence by the IH we have $Y x \in \overline{\mathscr{O}_{G}}$. Let $Y x=O \in \overline{\mathscr{C}_{G}}$. Then $M=\Xi l\left(\lambda x^{l} \cdot O\right) \in \overline{\mathscr{O}_{G}}$.

Case $\mathrm{G}_{\mathrm{L}} . \Gamma \vdash M$ is $\Gamma \vdash \mathrm{L}(\mathrm{G} X Y)$ as direct consequence of $\Gamma \vdash \mathrm{L} X, \quad \Gamma, X x \vdash \mathrm{L}(Y x)$ with $x \notin \mathrm{FV}(\Gamma, X, Y)$.

By the IH one has $L X \in \overline{\mathscr{C}_{G}}$, hence $X=l \in \mathscr{C}_{G}$. Again we may assume that $x=x^{l} \in \mathscr{T}_{G}^{l}$. Then $l x \in \overline{\mathscr{O}_{G}}$, hence by the IH we have $\mathrm{L}(Y x) \in \overline{\mathscr{C}_{G}}$. So $Y x=$ $\left.l_{1} \in \mathscr{C}_{G} . \mathrm{G} X Y=\mathrm{G} l\left(\lambda x^{l} . l_{1}\right)\right) \in \overline{\mathscr{D}_{G}}$ by Lemma 4.4. Hence $\mathrm{L}(\mathrm{G} X Y) \in \overline{\mathscr{G}_{G}} \subseteq \overline{\mathscr{O}_{G}}$.

The invariant $\mathscr{C}_{G}$ is more complicated than $\mathscr{C}$ and we cannot prove completeness directly using this grammar, as in in B3.11 for $\mathscr{T} \Xi$ relative to PRED. In fact we will use $\mathscr{C}_{G}$ in order to show in 4.12-4.21 that in each proof $\Gamma \vdash_{\mathscr{T}_{\mathrm{G}}} M$, where $\Gamma \subseteq \bar{O}_{G}$, all cut formulas can be eliminated. From this we get in Proposition 4.23 conservativity of $\mathscr{T} \mathrm{G}$ over $\mathscr{T} \Xi$

$$
\Gamma \subseteq \overline{\mathscr{O}}, M \in \overline{\mathscr{O}}, \Gamma \vdash_{\mathscr{T} \mathrm{G}} M \Rightarrow \Gamma \vdash_{\mathscr{T} \Xi} M
$$

and then completeness of $\mathscr{T G}$ for PRED is an immediate corollary of this proposition and Proposition B3.11.

\section{Cut elimination}

First we define the $\boldsymbol{\Xi}$-length $L_{\Xi}(O)$ for $O \in \overline{\mathscr{O}_{G}} \quad\left(\right.$ and $L_{\Xi}(l)$ for $\left.l \in \mathscr{C}_{G}\right)$ and state some lemma's, without the (trivial) proofs. 
4.12. Definition. ( $\Xi$-length $L_{\Xi}$ )

$$
\begin{aligned}
& L_{\Xi}\left(P t^{A_{1}}\right)=L_{\Xi}(\mathrm{L} l)=L_{\Xi}\left(A_{i} t^{A_{i}}\right)=0, \quad L_{\Xi}\left(\Xi l\left(\lambda x^{l} \cdot O\right)\right)=1+L_{\Xi}(l)+L_{\Xi}(O), \\
& L_{\Xi}\left(A_{i}\right)=0, \quad L_{\Xi}\left(\mathrm{G} l\left(\lambda x^{l} \cdot q\right)\right)=1+L_{\Xi}(l)+L_{\Xi}(q), \quad L_{\Xi}\left(\mathrm{K}_{p}\right)=L_{\Xi}(p) .
\end{aligned}
$$

4.13. Lemma. (i) $X, Y \in \mathscr{C}_{G} \cup \mathscr{O}_{G}, X \rightarrow_{\beta \eta} Y \Rightarrow L_{\Xi}(X)=L_{\Xi}(Y)$.

(ii) $X, Y \in \mathscr{C}_{G} \cup \mathscr{C}_{G}, X={ }_{\beta \eta} Y \Rightarrow L_{\Xi}(X)=L_{\Xi}(Y)$.

4.14. Definition. Let $M \in \overline{\mathscr{C}_{G}}, M={ }_{\beta \eta} O$. Then $L_{\Xi}(M)=L_{\Xi}(O)$.

4.15. Lemma. $L_{\Xi}\left(l t^{l}\right)=L_{\Xi}(l)$.

4.16. Lemma. $X \in \mathscr{C}_{G} \cup \overline{\mathscr{O}_{G}}, t^{l} \in \mathscr{T}_{G}^{l} \Rightarrow L_{\Xi}\left(X\left[x^{l}:=t^{l}\right]\right)=L_{\Xi}(X)$.

\subsection{Lemma.}

$$
\Xi X Y \in \overline{\mathscr{O}_{G}}, X V \in \overline{\mathscr{O}_{G}} \Rightarrow L_{\Xi}(X V)<L_{\Xi}(\Xi X Y) \& L_{\Xi}(Y V)<L_{\Xi}(\Xi X Y) .
$$

Proof. $\Xi X Y=\Xi l\left(\lambda x^{l} . O\right)$ and $V \in \overline{\mathscr{T}_{G}^{l}}$. Now use Lemma's 4.15 and 4.16.

4.18. Definition. A cut elimination is a proof reduction of the following form

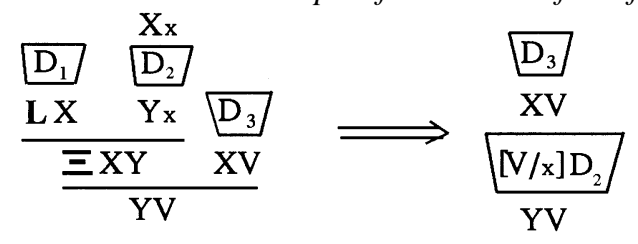

$\Xi X Y$ is called the cut formula.

4.19. Lemma. In a proof of $\Gamma \vdash_{\mathscr{T}_{\mathrm{G}}} M\left(\right.$ or $\left.\Gamma \vdash_{\mathscr{T} \Xi} M\right)$, where $\Gamma \subseteq \overline{\mathscr{O}_{G}}$, all cut formulas can be eliminated by the above proof reduction.

Proof. By induction on the maximal $\boldsymbol{\Xi}$-length of cut formulas in the proof of $\Gamma \vdash_{\mathscr{T}_{\mathrm{G}}} M$. Consider a first cut in the proof with a cut formula $\Xi X Y$ of maximal $\boldsymbol{\Xi}$-length. Eliminate this cut. Now new cut formulas may have been introduced at $X V$ and $Y V$, however by Lemma 4.17 these have shorter $\Xi$-length. In the same way all other cut formulas of maximal $\boldsymbol{\Xi}$-length can be eliminated and so by the induction hypothesis all cut formulas can be eliminated.

4.20. Remark. Because of Lemma 4.13, the above can easily be generalised to allow $\beta \eta$-equality steps (Eq-steps) as follows.

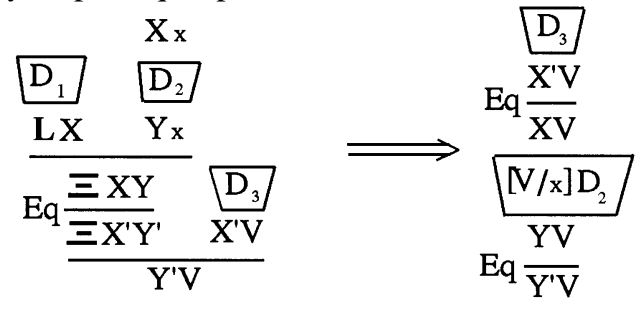


4.21. Remark. Similarly we can define cut elimination for $\mathscr{T P}$ as a proof reduction of the following form.

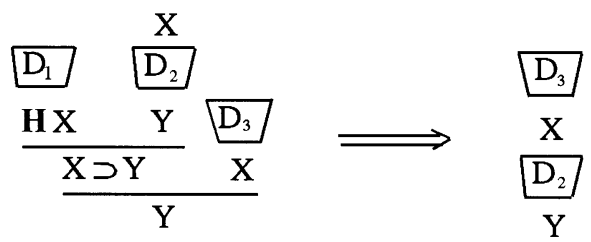

This is in fact a special case of Definition 4.18. ( Substitute $\mathrm{K} X$ for $X$ and $\mathrm{K} Y$ for $Y$ in Definition 4.18.) Also in a proof of $\Gamma \vdash_{\mathscr{P} \mathrm{P}} M$ where $\Gamma \subseteq \overline{\mathscr{O}_{G}}$ all cut formulas can be eliminated.

Now we need only one more lemma to derive conservativity of $\mathscr{T G}$ over $\mathscr{T} \Xi$ in Proposition 4.23 .

4.22. Lemma. Let $\Gamma \subseteq \overline{\mathcal{O}}$ and let $\Gamma \vdash_{\mathscr{T}_{\mathrm{G}}} N$ be a cut free proof. If the last applied rule in the proof is not the $\Xi_{\mathrm{i}}$-rule, nor the $\mathrm{G}_{\mathrm{L}}$-rule (modulo the $\beta \eta$-rule), then $N \in \bar{O}$.

Proof. The proof is by induction on $\Gamma \vdash N$. The two general cases are easy, so we only consider

Case $\Xi_{\mathrm{e}} . \Gamma \vdash N$ is $\Gamma \vdash Y V$ as direct consequence of $\Gamma \vdash \Xi X Y, \quad \Gamma \vdash X V$.

By the induction hypothesis for $\Gamma \vdash \Xi X Y$ we have $\Xi X Y \in \overline{\mathcal{O}}$. We distinguish two subcases

Subcase a). $\Xi X Y=\Xi A_{i}\left(\lambda x^{A_{i}} . O\right)$ with $O \in \overline{\mathscr{O}}$. The induction hypothesis for $\Gamma \vdash$ $A_{i} V$ yields $A_{i} V \in \overline{\mathscr{O}}$ and hence $V \in \overline{\mathscr{T}^{A_{i}}}$. So $N=Y V=O\left[x^{A_{i}}:=V\right] \in \overline{\mathscr{O}}$.

Subcase b). $\Xi X Y=\Xi(\mathrm{K} p)(\mathrm{K} O)$ with $O \in \overline{\mathcal{O}}$. Now $Y V=O \in \bar{O}$.

4.23. Proposition. $\mathscr{T G}$ is conservative over $\mathscr{T} \Xi$ in the following sense

$$
\Gamma \subseteq \overline{\mathscr{O}}, M \in \overline{\mathscr{O}}, \Gamma \vdash \mathscr{T}_{\mathrm{G}} M \Rightarrow \Gamma \vdash \mathscr{T} \boldsymbol{\Xi} M .
$$

Proof. By induction on the derivation of $\Gamma \vdash_{\mathscr{T}_{\mathrm{G}}} M$. We may assume that the proof is cut free. Again we only consider the three specific rules $\Xi_{\mathrm{e}}, \Xi_{\mathrm{i}}$ and $\mathrm{G}_{\mathrm{L}}$.

Case $\Xi_{\mathrm{e}} . \Gamma \vdash_{\mathscr{T}_{\mathrm{G}}} M$ is $\Gamma \vdash_{\mathscr{T}_{\mathrm{G}}} Y V$ as direct consequence of $\Gamma \vdash_{\mathscr{G}} \Xi X Y, \quad \Gamma \vdash_{\mathscr{T}_{\mathrm{G}}} X V$.

$\Xi X Y \in \overline{\mathscr{O}}$ by Lemma 4.22 , so by the IH we have $\Gamma \vdash \mathscr{T} \Xi \boldsymbol{\Xi} X Y$. Now $X=A_{i}$ or $X=\mathrm{K}_{1}$, so also $X V \in \bar{O}$ (use Lemma 4.22 for the case: $X=A_{i}$ ). Again by the IH we get $\Gamma \vdash_{\mathscr{T} \Xi} X V$. So $\Gamma \vdash_{\mathscr{T} \Xi} Y V$ by rule $\Xi_{\mathrm{e}}$.

Case $\Xi_{\mathrm{i}} \cdot \Gamma \vdash_{\mathscr{T}_{\mathrm{G}}} M$ is $\Gamma \vdash_{\mathscr{T}_{\mathrm{G}}} \boldsymbol{\Xi} X Y$ as direct consequence of $\Gamma \vdash_{\mathscr{T}_{\mathrm{G}}} \mathrm{L} X, \quad \Gamma, X x \vdash_{\mathscr{T}_{\mathrm{G}}} Y x$ with $x \notin \mathrm{FV}(\Gamma, X, Y)$.

$\Xi X Y \in \bar{O}$ so $X=A_{i}$ or $X=\mathrm{K} p_{1}$ and $Y x=O \in \overline{\mathscr{O}}$. So $L X \in \overline{\mathscr{O}}$, hence we have by 
the IH $\Gamma \vdash \mathscr{T} \Xi\llcorner X$. Now $\Gamma, X x \subseteq \overline{\mathscr{Q}}, Y x \in \overline{\mathscr{O}}$, so $\Gamma, X x \vdash \mathscr{T} \Xi Y x$ by the IH. Hence $\Gamma \vdash_{\mathscr{T} \Xi} \Xi X Y$.

Case $\mathrm{G}_{\mathrm{L}} . \Gamma \vdash_{\mathscr{T}_{\mathrm{G}}} M$ is $\Gamma \vdash_{\mathscr{T}_{\mathrm{G}}} \mathrm{L}(\mathrm{G} X Y)$ as direct consequence of $\Gamma \vdash_{\mathscr{T}_{\mathrm{G}}} \mathrm{L} X, \quad \Gamma, X x \vdash_{\mathscr{T}_{\mathrm{G}}} \mathrm{L}(Y x)$ with $x \notin \mathrm{FV}(\Gamma, X, Y)$.

$\mathrm{L}(\mathrm{G} X Y) \in \overline{\mathscr{O}}$ so $\mathrm{G} X Y=\mathrm{K} p$, where $p=\boldsymbol{\Xi} A_{i}\left(\lambda x^{A_{i}} \cdot p_{2}\right)$ or $p=\boldsymbol{\Xi}\left(\mathrm{K} p_{1}\right)\left(\mathrm{K} p_{2}\right)$ with $p_{1}, p_{2} \in \mathscr{P}$. Since $\mathrm{L} X \in \overline{\mathcal{O}}$, we have by the IH $\Gamma \vdash \mathscr{T} \Xi \mathrm{L} X$.

We may assume $x=x^{A_{i}}$. Now $(Y x)(z x)=p_{2}$. Also $\mathrm{L}(Y x) \in \overline{\mathscr{O}_{G}}$. So $Y x \in \mathscr{C}_{G}$. We can not have $Y x \in \mathscr{Q}_{G}$ by Lemma 4.7. So $Y x=\mathrm{K} p_{3}$ with $p_{3} \in \mathscr{P}_{G} \cdot p_{2}=(Y x)(z x)=$ $p_{3}$. So $Y x=\mathrm{K}_{2}$. Hence $\mathrm{L}(Y x)=\mathrm{H} p_{2} \in \bar{O}$ and the IH yields $\Gamma, X x \vdash_{\mathscr{T} \Xi} \mathrm{H} p_{2}$. If $p=\Xi A_{i}\left(\lambda x^{A_{i}} \cdot p_{2}\right)$ then we have $\Gamma, X x \vdash \mathscr{T} \Xi \mathrm{H}\left(\left(\lambda x^{A_{i}} \cdot p_{2}\right) x^{A_{i}}\right)$. So by rule $\Xi_{\mathrm{H}}$ we get $\Gamma \vdash_{\mathscr{T} \Xi} \mathrm{H}\left(\Xi A_{i}\left(\lambda x^{A_{i}} \cdot p_{2}\right)\right)$, i.e. $\Gamma \vdash_{\mathscr{T} \Xi} \mathrm{L}(\mathrm{G} X Y)$. If $p=\boldsymbol{\Xi}\left(\mathrm{K} p_{1}\right)\left(\mathrm{K}_{2}\right)$ then $\Gamma, p_{1} \vdash \mathscr{T} \Xi \mathrm{H} p_{2}$ where $x \notin \mathrm{FV}\left(\Gamma, p_{1}\right)$, so $x \notin \mathrm{FV}\left(p_{2}\right)$ by Proposition B3.10. So $\Gamma,\left(\mathrm{K} p_{1}\right) x \vdash_{\mathscr{T}} \Xi \mathrm{H}\left(\left(\mathrm{K} p_{2}\right) x\right)$ where $x \notin \mathrm{FV}\left(\mathrm{K} p_{2}\right)$, and therefore by rule $\Xi_{\mathrm{H}}$ we get $\Gamma \vdash_{\mathscr{T} \Xi} \mathrm{H}\left(\boldsymbol{\Xi}\left(\mathrm{K}_{p_{1}}\right)\left(\mathrm{K}_{p_{2}}\right)\right)$, i.e. $\Gamma \vdash_{\mathscr{T} \Xi} \mathrm{L}(\mathrm{G} X Y)$.

4.24. Proposition. (Completeness of $\mathscr{T G}$ for PRED)

$$
\Gamma_{s}^{1,+},[\Delta]^{1}, \Gamma(\Delta, \varphi) \vdash_{\mathscr{T}_{\mathrm{G}}}[\varphi]^{1} \Rightarrow \Delta \vdash_{\mathrm{PRED}} \varphi .
$$

Proof. This follows directly from the preceding proposition and Proposition B3.11.

\section{Conclusions and remarks}

In BBD (1993) and in this paper we proved soundness and completeness for the direct and indirect interpretations of propositional and predicate calculus into illative systems:

(i) $\Delta \vdash_{\mathrm{PROP}} \varphi \Leftrightarrow \forall$ closed $r\left[[\Delta]_{r}^{1}, \Gamma_{r}^{1}(\Delta, \varphi) \vdash_{\mathscr{T}}[\varphi]_{r}^{1}\right]$;

(ii) $\Delta \vdash_{\text {PRED }} \varphi \Leftrightarrow \forall$ closed $r\left[\Gamma_{r, s}^{1,+},[\Delta]_{r}^{1}, \Gamma(\Delta, \varphi) \vdash \mathscr{T} \Xi[\varphi]_{r}^{1}\right]$;

(iii) $\Delta \vdash_{\text {PROP }} \varphi \Leftrightarrow \forall$ closed $r \exists M\left[[\Delta]_{r}^{2}, \Gamma_{r}^{2}(\Delta, \varphi) \vdash_{\mathscr{F}}[\varphi]_{r}^{2} M\right]$;

(iv) $\Delta \vdash_{\mathrm{PRED}} \varphi \Leftrightarrow \forall \operatorname{closed} r \exists M\left[\Gamma_{r, s}^{2,+},[\Delta]_{r}^{2}, \Gamma(\Delta, \varphi) \vdash_{\mathscr{T} \mathrm{G}}[\varphi]_{r}^{2} M\right]$.

The proofs of soundness ( $\Rightarrow$ ) were simple for all 4 cases. The 2 completeness proofs for the first interpretation [ $]^{1}$ where given in BBD (1993) by specialising to $r=I$ whereas in the 2 completeness proofs for the second interpretation [ $]^{2}$ in the present paper we specialised to $r=\mathrm{K}$. One may wonder if these proofs could also be given by specialising to $r=\mathrm{I}$. This is indeed the case as is shown in DBB (1997).

Let us first compare the proof of completeness of $\mathscr{T G}$ for PRED in DBB (1997) with that in the present paper. In DBB (1997) we had to show

$$
\Gamma_{\mathrm{l}, s}^{2,+},[\Delta]_{l}^{2}, \Gamma(\Delta, \varphi) \vdash_{\mathscr{T}_{\mathrm{G}}}[\varphi]_{\mathrm{l}}^{2} M \Rightarrow \Delta \vdash_{\mathrm{PRED}} \varphi .
$$


This proof could not be reduced to a completeness proof for the first interpretation [ $]^{1}$. Hence the completeness could not follow from a conservativity result of $\mathscr{T}$ G over $\mathscr{T} \Xi$ like in Proposition 4.23. We defined an invariant $\mathscr{C}_{G}$ (modulo $\beta \eta$ equality) for derivations in $\mathscr{T} \mathrm{G}$. This invariant was similar to $\mathscr{C}_{G}$ of the present paper, but there were essential differences. For example in the present paper we can have $l \equiv \mathrm{K}\left(P t^{A_{1}}\right)$ with $\mathscr{T}_{G}^{l}=\Lambda^{s}(\boldsymbol{\Xi}, \mathrm{L})$, contrasted with $l \equiv\left(P t^{A_{1}}\right)$ and $\mathscr{T}_{G}^{l} \equiv \mathscr{T}_{G}^{l} \mid \mathscr{T}_{G}^{\mathrm{G} l_{1}\left(\lambda x^{l_{1}} . l\right)} \mathscr{T}_{G}^{l_{1}}$ in DBB (1997). Using this invariant we showed, like in Lemma 4.19, that in a proof of $\Gamma_{1, s}^{2,+},[\Delta]_{1}^{2}, \Gamma(\Delta, \varphi) \vdash_{\mathscr{T}_{\mathrm{G}}} N$ all cuts can be eliminated. Then we defined a second invariant $\mathscr{O}_{1}$ similar to $\mathscr{O}$ in B3.2 and using this invariant we proved directly completeness of $\mathscr{T G}$ for PRED

$$
\Gamma_{1, s}^{2,+},[\Delta]_{1}^{2}, \Gamma(\Delta, \varphi) \vdash_{\mathscr{T G}}[\varphi]_{1}^{2} M \Rightarrow \Delta \vdash_{\mathrm{PRED}} \varphi
$$

in a way similar to the proof of completeness of []$^{1}:$ PRED $\rightarrow \mathscr{T} \Xi$ in B3.2 B3.11. So we didn't have the conservativity result for $\mathscr{T} \mathrm{G}$ over $\mathscr{T} \Xi$

$$
\Gamma \subseteq \overline{\mathcal{O}}, M \in \overline{\mathscr{O}}, \Gamma \vdash_{\mathscr{T}} \mathrm{G} M \Rightarrow \Gamma \vdash \mathscr{T} \Xi M
$$

of Proposition 4.23.

Also the completeness proof of $\mathscr{T F}$ for PROP was proved in DBB (1997) directly i.e. without reducing it to a completeness proof for the first interpretation. Also in that case we didn't have the conservativity result for $\mathscr{T F}$ over $\mathscr{T P}$

$$
\Gamma \vdash \mathscr{F}_{\mathrm{F}} M \& \Gamma \subseteq \overline{\mathscr{O}_{0}} \Rightarrow \Gamma \vdash \mathscr{T}_{\mathrm{P}} M \& M \in \overline{\mathscr{C}_{0}}
$$

of Proposition 3.3.

5.1. Remark. In B4.1 we stated that it is possible to work with variants of the systems $\mathscr{T P}, \mathscr{T} \Xi, \mathscr{T F}$ and $\mathscr{T} \mathrm{G}$ based on $\beta$-conversion only. This still holds. It might seem that in the proof of 4.9 we used the $\eta$-axiom, but that is not the case, because by Definition 4.2 not only $f t^{A_{1}} \in \mathscr{T}_{G}^{A_{1}}$, but also $f \in \mathscr{T}_{G}{ }^{F_{1} A_{1}}$ and similarly $g \in \mathscr{T}_{G}{ }^{\mathrm{FA}}\left(\mathrm{FA} A_{2} A_{1}\right)$.

\section{References}

1. Barendregt H., Bunder M., Dekkers W. (1993) Systems of illative combinatory logic complete for first-order propositional and predicate calculus, J. Symb. Logic 58, 769-788

2. Dekkers W., Bunder M., Barendregt H. (1997) Completeness of the propositions-as-types interpretation of intuitionistic logic into illative combinatory logic, J. Symb. Logic (to appear)

3. Barendregt H. (1992) Lambda Calculi with Types, in: S. Abramski, D.M. Gabbay and T.S.E. Maibaum (eds.) Handbook of Logic in Computer Science, Vol II, Oxford University Press 